
3 Research Square
Preprints are preliminary reports that have not undergone peer review.
They should not be considered conclusive, used to inform clinical practice, or referenced by the media as validated information.

\title{
CCN1 gene polymorphisms associated with atrial septal defect susceptibility in Northwest Chinese population from different high-altitude areas
}

\author{
Wenke Yang \\ Lanzhou University School of Basic Medical Sciences \\ Jun Bai \\ Lanzhou University Second Hospital \\ Xinyu Song \\ Lanzhou University \\ Shasha Zhang \\ Lanzhou University \\ Nana Chen \\ Lanzhou University \\ Tao You \\ Gansu Provincial Hospital \\ Kang Yi \\ Gansu Provincial Hospital \\ Zhenglin Li \\ Lanzhou University \\ Dingxiong Xie \\ First People's Hospital of Lanzhou City \\ Xiaodong Xie ( $\nabla$ xdxie@lzu.edu.cn ) \\ Lanzhou University https://orcid.org/0000-0002-4895-0924
}

\section{Research Article}

Keywords: Congenital heart disease, CCN1 gene, Single nucleotide polymorphisms, High-altitude, Hypoxia

Posted Date: March 16th, 2021

DOI: https://doi.org/10.21203/rs.3.rs-277535/v1

License: (c) (7) This work is licensed under a Creative Commons Attribution 4.0 International License. Read Full License 


\section{Abstract}

High-altitude hypoxic environment exposure is considered as one of the risk factors for congenital heart disease (CHD), but the genetic factors involved are still unclear. CCN1, one of the synergistic molecules in the hypoxic response, is also an indispensable molecule in cardiac development. Considering that CCN1 may play an important role in the occurrence of $\mathrm{CHD}$ in high-altitude areas, we investigated the association between $\mathrm{CCN} 1$ polymorphisms and $\mathrm{CHD}$ susceptibility in Northwest Chinese population from different high-altitude areas. We conducted a case-control study with a total of $395 \mathrm{CHD}$ cases and 486 controls to evaluate the associations of $C C N 1$ polymorphisms with $\mathrm{CHD}$ risk. Our results showed that the protective alleles rs3753793-C $(\mathrm{OR}=0.59,95 \% \mathrm{Cl}=$ $0.42-0.81, P=0.001)$, rs2297141-A (OR $=0.66,95 \% \mathrm{Cl}=0.49-0.90, P=0.001)$ and $\mathrm{C}-\mathrm{A}$ haplotype of rs3753793-rs $2297141(\mathrm{OR}=0.58,95 \% \mathrm{Cl}=0.42-0.82, P=$ 0.002 ) were significantly associated with a decreased atrial septal defect (ASD) risk. Further subgroup analysis in different geography populations revealed robust association of SNP rs2297141 with ASD risk in a Han population residing in high-altitude of 2500-4287 m. We also found that the frequency of protective alleles was higher in high-altitude population, and the alleles were responsible for the difference of oxygen physiology related erythrocyte parameters in different high-altitude populations. rs3753793-C and rs2297141-A are likely related to high altitude and hypoxia adaptation, which may also be the reason for the association between $C C N 1$ polymorphism and ASD risk.

\section{Introduction}

Congenital heart disease (CHD) is a type of congenital malformation caused by abnormal cardiovascular development in embryonic period, and the incidence of live births is nearly 1\% (Cordell et al., 2013; Córdova-Palomera and Priest, 2019). It is estimated that ventricular septal defect (VSD), atrial septal defect (ASD), and atrioventricular septal defect (AVSD) are the common forms of congenital heart defect, accounting for $40 \%, 10 \%$, and $5 \%$ of $\mathrm{CHD}$, respectively (Hu et al., 2013; Xu et al., 2015; Sarısoy et al., 2018). As a complex genetic disease, environmental factors, genetic factors and their interaction are often used to explain the pathogenesis of CHD. Studies have shown that the prevalence of CHD in newborns increases with increasing altitude (Jackson, 1968; Patterson and Zhang, 2010; Zheng et al., 2013). Chronic high-altitude hypoxic environment exposure has been recognized as a trigger for this phenomenon (Jackson, 1968; Patterson and Zhang, 2010). Hypoxia in early pregnancy caused congenital cardiovascular abnormalities in $5.4 \%$ of the offspring of rats, compared with $3.2 \%$ in the control group (Haring, 1965). Maternal chronic hypoxic exposure increased cardiomyocyte apoptosis, promoted premature withdrawal from cell cycle and become terminal differentiated cardiomyocytes, and reduced the number of cardiomyocytes in fetal heart (Bae et al., 2003; Zhang, 2005; Patterson and Zhang, 2010; Ducsay et al., 2018). However, genetic factors related to hypoxic environment in the development of CHD are rarely reported.

Human CCN1 gene, also known as CYR61 or IGFBP10, is located on chromosome 1p22, which consisted of 4 introns and 5 exons. As a secreted heparinbinding extracellular matrix protein, CCN1 is involved in the formation of extracellular matrix structure, cell adhesion, proliferation, senescence and apoptosis (Mo et al., 2002; Mo and Lau, 2006; Kim et al., 2018). At embryonic heart development stage, decreased gelatinase activity and excessive cell apoptosis were observed in the atrioventricular cushion tissue of Ccn1-null mice, which may be caused by the disorder of extracellular matrix remodeling (Kim et al., 2018). Ccn1-null mice have AVSD or VSD phenotype, some Ccn1 haploinsufficient mice have ASD phenotype (Mo and Lau, 2006). Tetralogy of Fallot (TOF), a subtype of CHD, is often accompanied by hypoxemia (Therrien and Webb, 2003). In our previous work, we analyzed the gene expression profiles of TOF patients. CCN1 was down-regulated in myocardial tissue of TOF patients compared with healthy controls (Yang et al., 2019). Some studies have described that CCN1 as one of the synergistic molecules involved in hypoxic response, with mRNA stability and high expression under hypoxic conditions, while hypoxia exposure is one of the risk factors for CHD (Carraway et al., 2017; Ducsay et al., 2018; Fortenbery et al., 2018). Given the importance of CCN1 in cardiac development, it comes as no surprise that one low frequency variant p.L192L (rs9658586) and three rare variants p.S316C, p.R334W and p.R47W (rs148330006, rs9658587 and rs1346496644) in the CCN1 were identified to increase the risk of ASD (Perrot et al., 2015). The common variants rs954353 and rs3753793 were considered to be functional single nucleotide polymorphisms (SNP) that regulate the expression of the CCN1 gene and were associated with the risk of colorectal cancer and prostate cancer, respectively (Fernández-Rozadilla et al., 2010; Tao et al., 2013). However, the association of the CCN1 common SNP with the risk of CHD has not been reported to date.

In this work, three rare variants (rs148330006, rs9658587, rs1346496644) and four common tag SNPs (rs954353, rs3753793, rs2297141, rs9658584) of CCN1 gene were selected as candidate variants. Not only evaluate the association between candidate variants and the risk of CHD, but also assess the correlation between candidate variants and erythrocyte parameters reflecting oxygen physiology in populations at different altitudes (Crawford et al., 2006). This study may provide a new evidence from the perspective of gene-environment interaction to understand the high incidence of $\mathrm{CHD}$ in high-altitude population.

\section{Materials And Methods}

In silicoanalysis

As we described previously, mRNA expression profiles of TOF (GSE26125, GSE35776) were downloaded from the Gene Expression Omnibus (GEO) database, LIMMA package in R software is used for data normalization and differential expression analysis (Ritchie et al., 2015; Clough and Barrett, 2016; Yang et al., 2019). The average expression fold difference higher than 2 , the $P$ value less than 0.05 and the false discovery rate (FDR) less than 0.10 were regarded as the criterion for significant differential expression. Enrichment analysis of Gene Ontology biological process terms was performed on the KOBAS 3.0 website (Xie et al., 2011).

\section{Study subjects and sample collection}

For this case-control study, a total of 395 sporadic non-syndromic CHD patients and 486 unrelated healthy controls were recruited from the Gansu Provincial Hospital and the Second Hospital of Lanzhou University. There were 203 cases of VSD, 105 cases of ASD, 41 cases of TOF, 15 cases of AVSD, 15 cases of patent ductus arteriosus, 7 cases of pulmonary artery stenosis, 4 cases of coarctation of aorta, 3 cases of patent foramen ovale and 2 cases of double-outlet 
right ventricle. X-ray examination, electrocardiography and echocardiography were used for the diagnosis of CHD, and some cases were confirmed by surgery. The case group was composed of Han, Tibetan, Hui and Dongxiang groups in Northwest China, while the control group was composed of Han and Tibetan from Luqu county, Han from Lanzhou city, and other northwest population with or without recorded ethnic information. The altitude of Lanzhou city is 1500 $2000 \mathrm{~m}$, and Luqu county is located in the eastern edge of the Qinghai-Tibet Plateau, with an altitude of 2900-4287m. The clinical and demographic characteristics of study population are described in Table 1. Fresh peripheral blood samples were obtained from 95 of Luqu Tibetan, 98 of Luqu Han and 95 of Lanzhou Han, and the erythrocyte parameters include red blood cell (RBC) count and hemoglobin concentration (HGB) were measured using a Mindray BC6800 automated hematological analyzer (Mindray, Shenzhen, China). In addition, myocardial tissue samples were collected from 98 patients, soaked in RNA store solution and frozen at $-80^{\circ} \mathrm{C}$ for future use.

Written informed consent was obtained from all participants, and this study was approved by the Human Research Ethics Committees of Lanzhou University of Basic Medical Science (20160204).

Table 1

Demographic and clinical characteristics of the study population

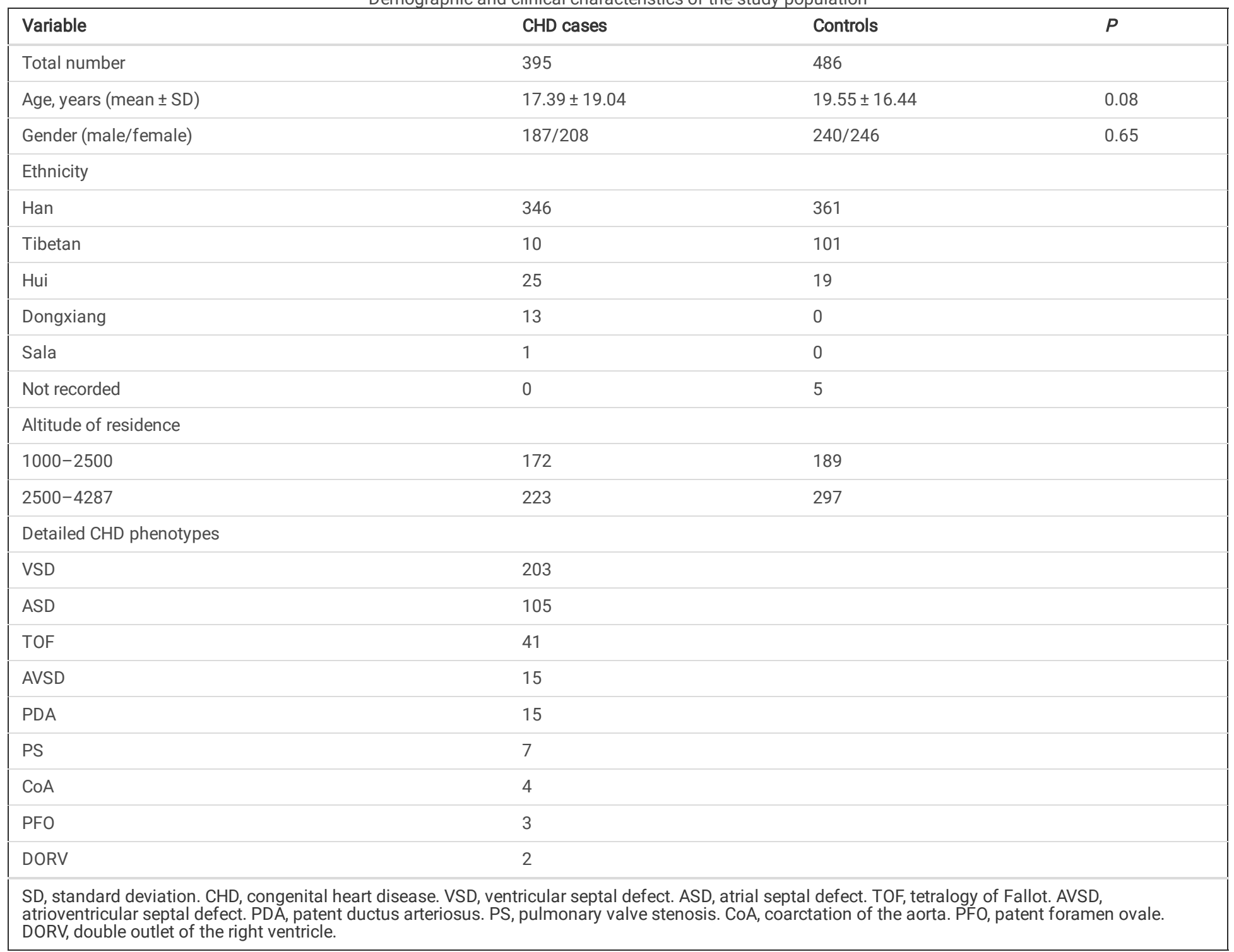

\section{DNA extraction and genotyping}

DNA extraction was performed with a Cwbio® Blood Genomic DNA Mini Kit according to the protocol (Cwbio, Beijing, China). SNP genotyping was performed using a multiplex ligation detection reaction SNP genotyping platform, as previously described (Yang et al., 2019). Sanger sequencing was used to verify genotyping results of about $5 \%$ randomly selected samples.

\section{RNA extraction and real-time quantitative reverse-transcription PCR}

The tissue samples were ground at low temperature in $1 \mathrm{~mL}$ TRIzol. Chloroform, isopropanol and alcohol were used for total RNA extraction. After purity and concentration detection, $1 \mu \mathrm{g}$ of total RNA was reverse transcribed to cDNA. QPCR SYBR® Green master mix and Real-Time PCR System were used to determine the expression of $C C N 1$ and internal control gene $\beta$-actin. The relative expression of $C C N 1$ in each sample was calculated by the $2^{-\triangle \Delta C t}$ method. The 
primer sequences of CCN1 were 5'-TGCCGCCTTGTGAAAGAAAC-3' (forward) and 5'-GAACCGCATCTTCACAGTCC-3' (reverse); the primers of $\beta$-actin were 5'GAAACTACCTTCAACTCCATC-3' (forward) and 5'-CTAGAAGCATTTGCGGTGGAC-3' (reverse).

\section{Statistical analysis}

The gene expression data were analyzed with the two-tailed unpaired Student t-test. For unequal variances, Welch's correction was followed. Categorical variables were evaluated by the chi-square $(\chi 2)$ test. Significant $P$-values were corrected for multiple testing with the Bonferroni correction. A corrected $P$-value less than 0.0125 was considered statistically significant for four available SNPs. Unconditional logistic regression analysis with adjusted for age and gender was also calculated. Multiple linear regression analysis was performed to evaluate the effect of gene polymorphism on erythrocyte parameters in different populations. Haplotype analysis and haplotype network construction were performed using SHEsis software and PopART software respectively (Li et al., 2009; Leigh and Bryant, 2015). The statistical analyses were performed using SPSS19.0 software package (SPSS, Chicago, IL, USA) and GraphPad Prism (GraphPad Prism Software, Inc.).

\section{Results}

\section{Decreased $C C N 1$ mRNA expression levels in TOF was identified by analyzing the GEO datasets}

A total of 32 non-syndromic TOF patients without the 22q11.2 deletion and 13 normal controls with expression data from two individual gene expression profiles (GSE26125, GSE35776) in the GEO database were available for differentially expressed genes analysis. CCN1 as a shared down-regulated differentially expressed gene in TOF patients of GSE26125 and GSE35776 (Fig. 1). Subsequent gene function enrichment analysis demonstrated that CCN1 was involved in multiple heart development biological processes, such as atrial septum morphogenesis (G0:0060413, $P=0.007)$, atrioventricular valve morphogenesis (GO:0003181, $P=0.008)$, cardiac atrium morphogenesis (G0:0003209, $P=0.013)$, cardiac septum development (G0:0003279, $P=0.040)$, cardiac ventricle development (G0:0003231, $P=0.050)$, heart valve development (G0:0003170, $P=0.017$ ), ventricular septum development (G0:0003281, $P=$ $0.026)$ and apoptotic process involved in heart morphogenesis (G0:0003278, $P=0.003$ ).

\section{CCN1 gene polymorphism and CHD risk}

Three rare variants (rs148330006, rs9658587, rs1346496644) were no polymorphism in all tested samples and were not available for subsequent statistical analysis. The genotype and each genetic model frequencies of four common SNPs (rs954353, rs3753793, rs2297141, rs9658584) are listed in Table 2. The genotype distributions of all SNP in controls were in accordance with the Hardy-Weinberg equilibrium. In our study population, the genotype AC of rs3753793 was associated with a significantly decreased $\mathrm{CHD}$ risk (AC vs. $\mathrm{AA}: \mathrm{OR}=0.66,95 \% \mathrm{Cl}=0.50-0.89, P=0.006 ; \mathrm{AC}+\mathrm{CC}$ vs. $\mathrm{AA}$ : OR $=0.68,95 \% \mathrm{Cl}=0.51-0.89, P$ $=0.006)$. Further analysis conducted in subclassification groups of $\mathrm{CHD}$, association were observed in $\mathrm{ASD}(\mathrm{C}$ vs. $\mathrm{A}: \mathrm{OR}=0.59,95 \% \mathrm{Cl}=0.42-0.81, P=0.001$; $\mathrm{AC}$ vs. $\mathrm{AA}: \mathrm{OR}=0.54,95 \% \mathrm{Cl}=0.35-0.85, P=0.008 ; \mathrm{CC}$ vs. $\mathrm{AA}: \mathrm{OR}=0.34,95 \% \mathrm{Cl}=0.15-0.74, P=0.006 ; \mathrm{AC}+\mathrm{CC}$ vs. $\mathrm{AA}: \mathrm{OR}=0.50,95 \% \mathrm{Cl}=0.32-0.76, P=$ 0.001 ) and AVSD (AC vs. AA: $\mathrm{OR}=0.13,95 \% \mathrm{Cl}=0.03-0.58, P=0.002 ; \mathrm{AC}+\mathrm{CC}$ vs. $\mathrm{AA}: \mathrm{OR}=0.24,95 \% \mathrm{Cl}=0.08-0.72, P=0.010$ ), but not in VSD and TOF (Table 2). For rs2297141, the association between rs2297141 polymorphism and the incidence of ASD (A vs. G: OR =0.66, 95\% Cl =0.49-0.90, $P=0.008 ; \mathrm{AA}$ vs. $\mathrm{GG}$ : $\mathrm{OR}=0.37,95 \% \mathrm{Cl}=0.19-0.74, P=0.004 ; \mathrm{AA}$ vs. $\mathrm{GA}+\mathrm{GG}$ : $\mathrm{OR}=0.41,95 \% \mathrm{Cl}=0.22-0.76, P=0.004)$ was presented. No association was observed in VSD, TOF and AVSD (Table 2).

All the ASDs we included were Chinese Han ethnic. Considering different geography, different ethnic populations have different genetic backgrounds. Here, we further analyzed the association between $C C N 1$ gene polymorphism and ASD risk in Han population at different altitudes of residence. No statistical significance difference between altitude 2500-4287 m group and altitude 1000-2500 m group in Han population was apparent $(P>0.05)($ Supplementary Table S1). In Han population, significant association with the ASD risk was observed for rs 2297141 under the recessive genetic model in both overall group ( $P$ $=0.010)$ and altitude 2500-4287 m subgroup $(P=0.011)$ (Table 3). In contrast, a significant association between CCN1 gene polymorphism and CHD or ASD risk was not found in Tibetan or altitude 1000-2500 m subgroup of Han populations (Table 3 and Supplementary Table S2).

Further haplotype analysis showed that C-A haplotype in rs3753793-rs2297141 had a significantly lower risk for $\mathrm{CHD}(\mathrm{OR}=0.79,95 \% \mathrm{Cl}=0.64-0.97, P=$ $0.021)$, especially for ASD $(O R=0.58,95 \% \mathrm{Cl}=0.42-0.82, P=0.002)$. The frequency of C-A haplotype was with a borderline statistical difference between $\mathrm{CHD}$ cases and controls in Tibetan populations $(\mathrm{OR}=0.38,95 \% \mathrm{Cl}=0.14-1.00, P=0.052)$.

\section{Association of CCN1 gene polymorphism with CCN1 mRNA expression}

DNA and RNA were extracted from 98 myocardial tissue samples. DNA was used to detect $C C N 1$ gene polymorphism, and RNA was used to detect CCN1 mRNA expression. The gene expression corresponding to each genotype was used to measure the correlation between $C C N 1$ gene polymorphism and $C C N 1$ mRNA expression. Our results indicated that there was no significant correlation between the four SNP polymorphisms and CCN1 mRNA expression (Fig. 2). However, both the $\mathrm{C}$ allele of rs3753793 and the A allele of rs2297141, which reduced the risk of ASD, showed slightly higher median value of CCN1 expression in the carriers, and there was no significant statistical difference, the $P$-values in the dominant model were 0.26 and 0.41 , respectively.

\section{The association between CCN1 gene polymorphism and erythrocyte parameters in different geographical and ethnic populations}

Fresh peripheral blood samples were obtained from Luqu Tibetan, Luqu Han and Lanzhou Han populations for RBC, HGB assays and DNA extraction. The distribution differences of CCN1 gene polymorphism in the above three populations were shown in Supplementary Tables S3, S4 and S5. Compared with Lanzhou Han population, the $C$ allele of rs3753793 $(P<0.05)$ and the C-A haplotype of rs3753793-rs2297141 $(P=0.047)$ were specific to Luqu Tibetan population. The A allele of rs2297141 $(P<0.05)$ was specific to Luqu Tibetan compared to Luqu Han. However, these observed statistical differences were not stay significant after Bonferroni correction. We also downloaded the data of three Chinese populations (CHB, CHS, CDX) from 1000 Genomes Project, and 
further analyzed the haplotype network of the six Chinese populations (Fig. 3). C-A haplotype showed a high proportion in high-altitude Lanzhou Han, Luqu Han and Luqu Tibetan populations, especially in Luqu populations, this observation may be related to the altitude of the population living environment.

When considering the erythrocyte parameters, the RBC count of Lanzhou Han, Luqu Han and Luqu Tibetan were $4.918 \pm 0.495\left(\times 10^{12} / \mathrm{L}\right), 4.891 \pm 0.444$ $\left(\times 10^{12} / \mathrm{L}\right)$ and $4.871 \pm 0.500\left(\times 10^{12} / \mathrm{L}\right)$, respectively, and there was no obvious statistical significance among the groups. The HGB of Lanzhou Han, Luqu Han and Luqu Tibetan were $151.9 \pm 18.40(\mathrm{~g} / \mathrm{L}), 143.9 \pm 15.75(\mathrm{~g} / \mathrm{L})$ and $139.5 \pm 17.33(\mathrm{~g} / \mathrm{L})$, respectively. Compared with Lanzhou Han, the HGB of Luqu Han $(P=$ $0.002)$ and Luqu Tibetan $(P<0.001)$ were significantly different. The results of multiple linear regression analysis showed that there was a significant difference in the RBC (rs3753793, AC+CC vs. AA: $\mathrm{BETA}=0.142,95 \% \mathrm{Cl}=0.035-0.249$, Pcor. $=0.010 ; \mathrm{AC}$ vs. $\mathrm{AA}: \mathrm{BETA}=0.147,95 \% \mathrm{Cl}=0.035-0.258, P c o r .=$ 0.011. rs2297141, GA+AA vs. GG: BETA $=-0.175,95 \% \mathrm{Cl}=-0.305-0.045$, Pcor. $=0.009)$ or HGB (rs3753793, AC vs. AA: $\mathrm{BETA}=4.222,95 \% \mathrm{Cl}=0.057-8.387$, Pcor. $=0.048$ ) between Luqu Tibetan and Lanzhou Han for rs3753793 under the dominant and heterozygous genetic models and for rs2297141 under the dominant genetic model after adjustment for age and gender (Table 4).

\section{Discussion}

The etiology of sporadic non-syndromic CHD is currently widely accepted by environmental factors, genetic factors and their interaction (Vecoli et al., 2014; Baldacci et al., 2018). Chronic hypoxic exposure is considered to be the main factor for the high incidence of CHD in plateau area, but the genetic factors involved are still unclear and need to be evaluated (Jackson, 1968; Patterson and Zhang, 2010; Zheng et al., 2013). Studies have shown that CCN1 is indispensable for cushion tissue development at embryonic heart development stage, the VSD, ASD, AVSD phenotypes were found in CCN1 deficient mice (Mo and Lau, 2006). In our previous study, we identified that CCN1 expressed at low levels in TOF, and the enrichment of CCN1 gene function showed that CCN1 plays an important role in heart development (Yang et al., 2019). Four variants of CCN1, p.L192L (rs9658586), p.S316C (rs148330006), p.R334W (rs9658587) and p.R47W (rs1346496644) were also described to be associated with ASD (Perrot et al., 2015). In the present study, we investigated the association of four detected SNP of CCN1 with CHD susceptibility in a Northwest Chinese population. There was significant difference in genotype distribution of rs3753793 between CHD case group and healthy control group, with the AC genotype of rs 3753793 or the dominant genetic model of rs3753793 being associated with a decreased risk of CHD. In subgroup analyses of CHD type, rs3753793 was associated with the risk of ASD and AVSD, rs2297141 was associated with the risk of ASD, and the C-A haplotype of rs3753793-rs2297141 was also associated with ASD. No association was observed between the other two SNPs and any type of CHD risk. This observed correlation in a specific subtype of CHD may be explained by the etiological heterogeneity of different CHD subtypes, different anatomical locations of heart with distinct differentiation processes. For example, AMHC1 and FOXA2 are specific developmental regulators for atrial and ventricular, respectively (Yutzey et al., 1994; Bardot et al., 2017). Previous studies have also reported that gene deficient or missense mutations of CCN1 was associated with the ASD phenotype, which seems to indicate that CCN1 may have a special role in the development of atrial septum (Mo and Lau, 2006; Perrot et al., 2015).

Previous studies have shown that rs 954353 and rs3753793 may be located in the regulatory regions and affect CCN1 gene expression (Fernández-Rozadilla et al., 2010; Tao et al., 2013; Ali et al., 2015). Tao et al. believed that the CCN1 expression of the rs3753793 AA genotype is 1.49 times higher than that of AC/CC genotypes in prostate cancer tissues (Tao et al., 2013). Finding reported by Ali et al. is consistent with this (Ali et al., 2015). Patients with fracture nonunion who carried the AA genotype of rs3753793 showed 1.36 times higher CCN1 mRNA levels in blood samples than individuals carrying the AA/AC genotypes (Ali et al., 2015). In our present work, the expression levels of CCN1 and a potential correlation with four SNPs genotypes were evaluated, but no significant difference was found. However, the carriers of ASD-protective allele C of rs3753793 or A of rs2297141 have slightly higher median value of CCN1 expression levels in the myocardial tissue, which is inconsistent with previous studies. This is likely to be the reason that there are specific expression regulation patterns in different tissues, which needs further study (Ye et al., 2018). In addition, SNPs that were not significantly related to the expression levels of $C C N 1$ but were related to ASD susceptibility may have the following two reasons. First, the bias caused by the small size of tissue samples to detect the expression levels of $C C N 1$. Second, combined with previous reports, the relationship between $C C N 1$ gene polymorphism and expression levels was inconsistent in different tissues. However, the relationship in different anatomical locations of the heart (atrial septum, interventricular septum, atrium, ventricle) was not completely determined. These two hypotheses still need to be verified in well-designed studies with large sample sizes.

There are many ethnic populations living in northwest China for generations, and the living environment has a wide range of altitude. Different ethnic groups have different genetic backgrounds or living environments (Li et al., 2018). In the subgroup analysis, rs2297141 showed a significant correlation with CHD in both overall group and altitude 2500-4287 m subgroup of Han populations, but not in Tibetan or altitude 1000-2500 m subgroup of Han populations. The reason may be due to the small sample size in ethnic subgroups, or the $C C N 1$ gene polymorphism is distributed differently in different geographical and ethnic populations. Therefore, we further analyzed the distribution of CCN1 gene polymorphism in Luqu Tibetan, Luqu Han and Lan Zhou Han populations. The results showed that the $\mathrm{C}$ allele of rs3753793, the A allele of rs2297141, the C-A haplotype of rs3753793-rs2297141, which reduced the risk of ASD, have a significantly higher frequency in Luqu Tibetan population than in Luqu Han and Lanzhou Han populations. Luqu County lies on the eastern margin of the Qinghai-Tibet Plateau, with an altitude of 2900-4287m. Lanzhou City has an altitude of 1500-2000m, Xishuangbanna City has an altitude of 600-1600m; Southern China has an altitude of 5-350m; Beijing has an altitude of 28-107 m. The CCN1 gene polymorphism data of Han Chinese in Beijing (CHB), Southern Han Chinese (CHS) and Chinese Dai in Xishuangbanna (CDX) in the 1000 Genomes Project were combined for haplotype network analysis, it still showed that C-A haplotype accounted for the highest proportion in Luqu Tibetan population, and the proportion of high-altitude population was higher than that of lowaltitude population. These results suggest that the C allele of rs3753793, the A allele of rs2297141, the C-A haplotype of rs3753793-rs2297141 may be related to high-altitude adaptation, which in turn affects the risk of ASD in different geographical and ethnic populations.

High-altitude hypoxic environment was one of the reasons for the high incidence of CHD in the plateau area (Zheng et al., 2013; Ducsay et al., 2018). CCN1 was described to be one of the synergistic molecules involved in the hypoxic response (Carraway et al., 2017; Fortenbery et al., 2018). Although the molecular mechanism is not clear, considering that hypoxia may be the messenger between CCN1 and ASD, the relationship between CCN1 gene polymorphism and oxygen-related physiology is worth exploring. Oxygen carrying hemoglobin in red blood cells is the key molecule for tissues and organs to obtain oxygen. The

Page 5/15 
RBC and HGB of Luqu Tibetan, Luqu Han and Lanzhou Han populations were detected in this study. The RBC and HGB of Luqu population are lower than that of Lanzhou population, this observation is consistent with the report from Li et al (Li et al., 2018). For the people who adapt to high-altitude hypoxia, low levels of RBC and HGB have a high oxygen affinity. The Luqu Tibetan population has lower RBC and HGB than the Luqu Han population. The differences of evolutionary pressure and genetic background between Luqu Tibetan population and Luqu Han population may be responsible for this phenomenon (Li et al., 2018). This also showed that the samples we included were representative of the different populations. The polymorphisms of rs 3753793 and rs 2297141 in CCN1 gene were also associated with the differences of RBC or HGB in Luqu Tibetan and Lanzhou Han population. These results further suggested that the $C C N 1$ gene polymorphism may be related to high-altitude and hypoxia adaptation. The difference in hypoxic response is probably the reason for the association between $C C N 1$ gene polymorphism and ASD risk in different geographical and ethnic populations.

In the current work, we tried to analyze the interaction between genetic factors and hypoxia environmental factor in the development of CHD. TOF expression profile data analysis, gene function enrichment analysis and literature review were used to identify genes related to both hypoxia response and cardiac development. CCN1 was selected as a candidate gene, and we investigated the association between CCN1 gene polymorphism and CHD risk in in different geographical and ethnic populations at different altitudes. We also analyzed the correlation between gene polymorphism and oxygen physiology-related erythrocyte parameters in different altitude populations. However, the present work has several limitations. First, a relatively small number of study population was included, especially in subgroup analysis, which may weaken the statistical power of our analysis. A large sample size is necessary to reveal a strong conclusion. Second, the population we include was geographically limited. The applicability of our results should be verified in other populations. Third, as we stated earlier, the sample size for measuring $C C N 1$ expression in myocardial tissue was small, and the anatomical location of the tissue source may also be worth considering.

In conclusion, we found that rs3753793 and rs2297141 polymorphisms of CCN1 gene are associated with ASD susceptibility in a Northwest Chinese population. There were ethnic and geographical differences in this correlation, and the frequency of protective alleles was higher in high-altitude population. Protective alleles may affect hypoxic response, participate in the process of high-altitude adaptation, and may also be the reason for reducing ASD risk. Our findings might provide some new insight into the etiology of non-syndromic sporadic CHD in high-altitude population from the perspective of geneenvironment interaction. However, our results need further validation in a larger population, and the specific molecular mechanism of CCN1 gene which regulates hypoxia response still needs further investigation.

\section{Declarations}

Funding This work was supported by the National Natural Science Foundation of China (grant numbers 31670379 and 31660112 ) and the Program for Innovation and Entrepreneurship of Lanzhou University Students (grant number 20190060166).

Acknowledgments We express our appreciation to the original DNA donors who made this study possible.

Author contributions $X X, D X$ and WY designed the study. WY, JB, TY and KY enrolled the samples and clinical data. WY, JB, XS and SZ performed the experiments. WY, JB, XS, SZ, NC and ZL participated in data analysis. WY drafted the manuscript. XX revised the manuscript critically. All authors read and approved the final manuscript.

Data availability The datasets generated for this study are available from the corresponding author on reasonable request.

\section{Compliance with ethical standards}

Conflict of interest The authors declare that the research was conducted in the absence of any commercial or financial relationships that could be construed as a potential conflict of interest.

Ethics approval This study was approved by the Ethics Committees of Lanzhou University of Basic Medical Science (20160204). The patients/participants provided their written informed consent to participate in this study.

Informed consent Written informed consent to participate in this study was provided by the participants or participants' legal guardian.

\section{References}

Ali, S., Hussain, S.R., Singh, A., Kumar, V., Walliullah, S., Rizvi, N., et al. (2015). Study of Cysteine-Rich Protein 61 Genetic Polymorphism in Predisposition to Fracture Nonunion: A Case Control. Genet Res Int 2015: 754872

Bae, S., Xiao, Y., Li, G., Casiano, C.A., and Zhang, L. (2003). Effect of maternal chronic hypoxic exposure during gestation on apoptosis in fetal rat heart. Am J Physiol Heart Circ Physiol 285: 983-990

Baldacci, S., Gorini, F., Santoro, M., Pierini, A., Minichilli, F., and Bianchi, F. (2018). Environmental and individual exposure and the risk of congenital anomalies: a review of recent epidemiological evidence. Epidemiol Prev 42: 1-34

Bardot, E., Calderon, D., Santoriello, F., Han, S., Cheung, K., Jadhav, B., et al. (2017). Foxa2 identifies a cardiac progenitor population with ventricular differentiation potential. Nat Commun 8: 14428

Carraway, K.R., Johnson, E.M., Kauffmann, T.C., Fry, N.J., and Mansfield, K.D. (2017). Hypoxia and Hypoglycemia synergistically regulate mRNA stability. RNA Biol 14: 938-951 
Clough, E., and Barrett, T. (2016). The Gene Expression Omnibus Database. Methods Mol Biol 1418: 93-110

Cordell, H.J., Töpf, A., Mamasoula, C., Postma, A.V., Bentham, J., Zelenika, D., et al. (2013). Genome-wide association study identifies loci on $12 q 24$ and $13 q 32$ associated with tetralogy of Fallot. Hum Mol Genet 22: 1473-1481

Córdova-Palomera, A., and Priest, J.R. (2019). Association between the $4 \mathrm{p} 16$ genomic locus and different types of congenital heart disease: results from adult survivors in the UK Biobank. Sci Rep 9: 16515

Crawford, J.H., Isbell, T.S., Huang, Z., Shiva, S., Chacko, B.K., Schechter, A.N., et al. (2006). Hypoxia, red blood cells, and nitrite regulate NO-dependent hypoxic vasodilation. Blood 107: 566-574

Ducsay, C.A., Goyal, R., Pearce, W.J., Wilson, S., Hu, X.Q., and Zhang, L. (2018). Gestational Hypoxia and Developmental Plasticity. Physiol Rev 98: $1241-1334$

Fernández-Rozadilla, C., Tarrío, R., Clofent, J., de Castro, L., Brea-Fernández, A., Bessa, X., et al. (2010). Colorectal cancer susceptibility quantitative trait loci in mice as a novel approach to detect low-penetrance variants in humans: a two-stage case-control study. Cancer Epidemiol Biomarkers Prev 19: 619-623

Fortenbery, G.W., Sarathy, B., Carraway, K.R., and Mansfield, K.D. (2018). Hypoxic stabilization of mRNA is HIF-independent but requires mtROS. Cell Mol Biol Lett 23: 48

Haring, O.M. (1965). Effects of prenatal hypoxia on the cardiovascular system in the rat. Arch Pathol 80: 351-356

Hu, C., Huang, S., Xu, Z., and Huang, J. (2013). Hybrid treatment of a dislocated atrial septal occluder device at the bifurcation of the left and right common iliac artery. Interact Cardiovasc Thorac Surg 16: 701-702

Jackson, B.T. (1968). The pathogenesis of congenital cardiovascular anomalies. N Engl J Med 279: 80-89

Kim, K.H., Won, J.H., Cheng, N., and Lau, L.F. (2018). The matricellular protein CCN1 in tissue injury repair. J Cell Commun Signal 12: $273-279$

Leigh, J., and Bryant, D. (2015). PopART: Full-Feature Software for Haplotype Network Construction. Methods in Ecology and Evolution 6: $1110-1116$

Li, C., Li, X., Liu, J., Fan, X., You, G., Zhao, L., et al. (2018). Investigation of the differences between the Tibetan and Han populations in the hemoglobin-oxygen affinity of red blood cells and in the adaptation to high-altitude environments. Hematology 23: 309-313

Li, Z., Zhang, Z., He, Z., Tang, W., Li, T., Zeng, Z., et al. (2009). A partition-ligation-combination-subdivision EM algorithm for haplotype inference with multiallelic markers: update of the SHEsis (http://analysis.bio-x.cn). Cell Res 19: 519-523

Mo, F.E., and Lau, L.F. (2006). The matricellular protein CCN1 is essential for cardiac development. Circ Res 99: 961-969

Mo, F.E., Muntean, A.G., Chen, C.C., Stolz, D.B., Watkins, S.C., and Lau, L.F. (2002). CYR61 (CCN1) is essential for placental development and vascular integrity. Mol Cell Biol 22: 8709-8720

Patterson, A.J., and Zhang, L. (2010). Hypoxia and fetal heart development. Curr Mol Med 10: 653-666

Perrot, A., Schmitt, K.R., Roth, E.M., Stiller, B., Posch, M.G., Browne, E.N., et al. (2015). CCN1 mutation is associated with atrial septal defect. Pediatr Cardiol 36: 295-299

Ritchie, M.E., Phipson, B., Wu, D., Hu, Y., Law, C.W., Shi, W., et al. (2015). limma powers differential expression analyses for RNA-sequencing and microarray studies. Nucleic Acids Res 43: e47

Sarısoy, Ö., Ayabakan, C., Tokel, K., Özkan, M., Türköz, R., and Aşlamacı, S. (2018). Long-term outcomes in patients who underwent surgical correction for atrioventricular septal defect. Anatol J Cardiol 20: 229-234

Tao, L., Chen, J., Zhou, H., Qin, C., Li, P., Cao, Q., et al. (2013). A functional polymorphism in the CYR61 (IGFBP10) gene is associated with prostate cancer risk. Prostate Cancer Prostatic Dis 16: 95-100

Therrien, J., and Webb, G. (2003). Clinical update on adults with congenital heart disease. Lancet 362: 1305-1313

Vecoli, C., Pulignani, S., Foffa, I., and Andreassi, M.G. (2014). Congenital heart disease: the crossroads of genetics, epigenetics and environment. Curr Genomics 15: 390-399

Xie, C., Mao, X., Huang, J., Ding, Y., Wu, J., Dong, S., et al. (2011). KOBAS 2.0: a web server for annotation and identification of enriched pathways and diseases. Nucleic Acids Res 39: 316-322

Xu, Y., Liu, J., Wang, J., Liu, M., Xu, H., and Yang, S. (2015). Factors influencing the spontaneous closure of ventricular septal defect in infants. Int J Clin Exp Pathol 8: 5614-5623

Yang, W., Yi, K., Yu, H., Ding, Y., Li, D., Wei, Y., et al. (2019). Correlation between pri-miR-124 (rs531564) polymorphism and congenital heart disease susceptibility in Chinese population at two different altitudes: a case-control and in silico study. Environ Sci Pollut Res Int 26: $21983-21992$ 
Ye, B., Liu, B., Hao, L., Zhu, X., Yang, L., Wang, S., et al. (2018). Klf4 glutamylation is required for cell reprogramming and early embryonic development in mice. Nat Commun 9: 1261

Yutzey, K.E., Rhee, J.T., and Bader, D. (1994). Expression of the atrial-specific myosin heavy chain AMHC1 and the establishment of anteroposterior polarity in the developing chicken heart. Development 120: 871-883

Zhang, L. (2005). Prenatal hypoxia and cardiac programming. J Soc Gynecol Investig 12: 2-13

Zheng, J.Y., Tian, H.T., Zhu, Z.M., Li, B., Han, L., Jiang, S.L., et al. (2013). Prevalence of symptomatic congenital heart disease in Tibetan school children. Am J Cardiol 112: 1468-1470

\section{Tables}

Table 1 Demographic and clinical characteristics of the study population

\begin{tabular}{|c|c|c|c|}
\hline Variable & CHD cases & Controls & $P$ \\
\hline Total number & 395 & 486 & \\
\hline Age, years (mean $\pm S D)$ & $17.39 \pm 19.04$ & $19.55 \pm 16.44$ & 0.08 \\
\hline Gender (male/female) & $187 / 208$ & $240 / 246$ & 0.65 \\
\hline \multicolumn{4}{|l|}{ Ethnicity } \\
\hline Han & 346 & 361 & \\
\hline Tibetan & 10 & 101 & \\
\hline Hui & 25 & 19 & \\
\hline Dongxiang & 13 & 0 & \\
\hline Sala & 1 & 0 & \\
\hline Not recorded & 0 & 5 & \\
\hline \multicolumn{4}{|l|}{ Altitude of residence } \\
\hline $1000-2500$ & 172 & 189 & \\
\hline $2500-4287$ & 223 & 297 & \\
\hline \multicolumn{4}{|l|}{ Detailed CHD phenotypes } \\
\hline VSD & 203 & & \\
\hline ASD & 105 & & \\
\hline TOF & 41 & & \\
\hline AVSD & 15 & & \\
\hline PDA & 15 & & \\
\hline PS & 7 & & \\
\hline $\mathrm{CoA}$ & 4 & & \\
\hline $\mathrm{PFO}$ & 3 & & \\
\hline DORV & 2 & & \\
\hline
\end{tabular}

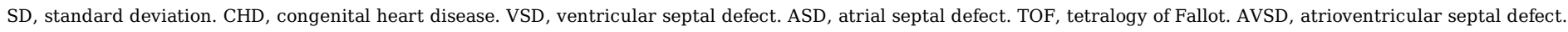
PDA, patent ductus arteriosus. PS, pulmonary valve stenosis. CoA, coarctation of the aorta. PFO, patent foramen ovale. DORV, double outlet of the right ventricle. 


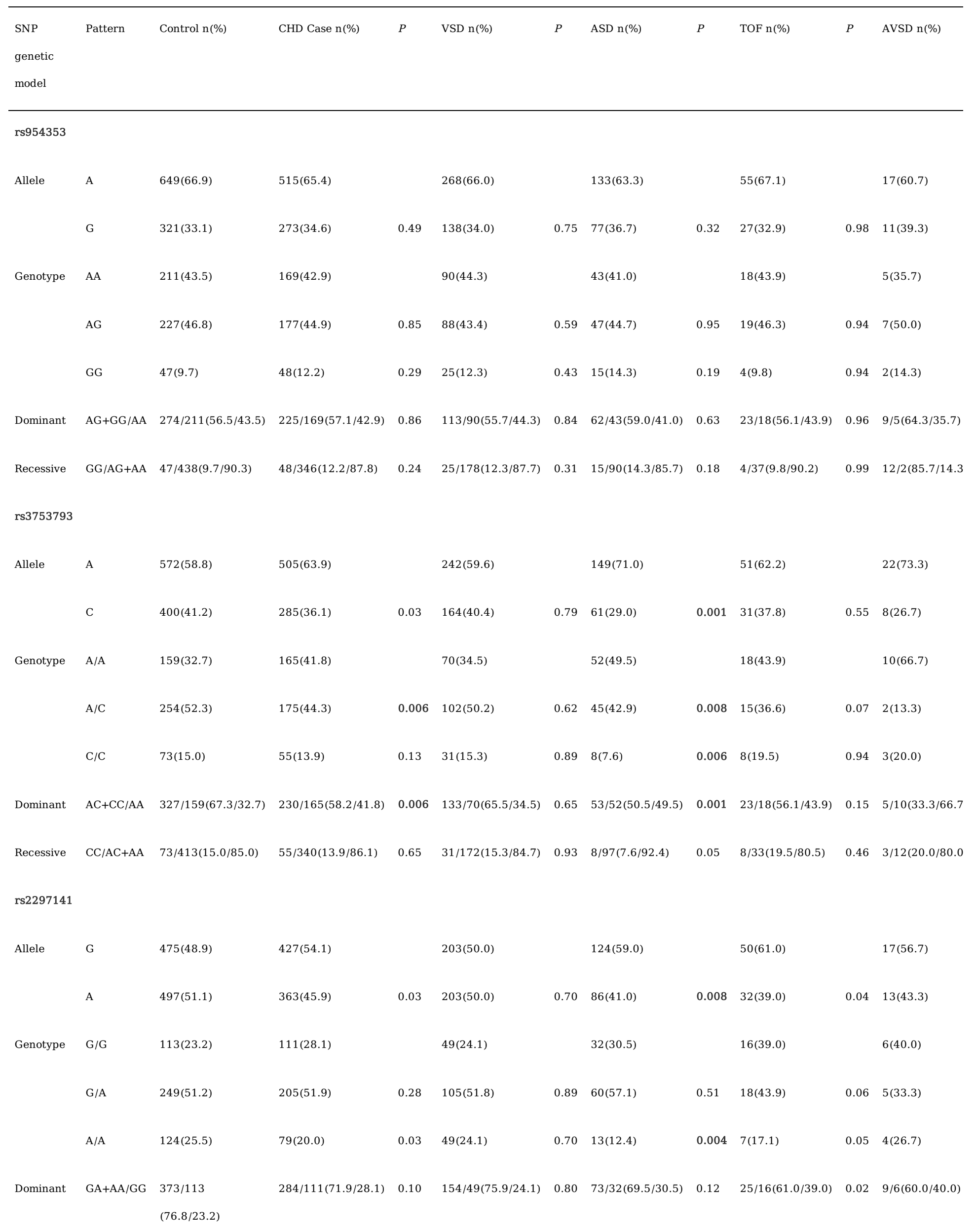




\begin{tabular}{|c|c|c|c|c|c|c|c|c|c|c|c|}
\hline Recessive & $\mathrm{AA} / \mathrm{GA}+\mathrm{GG}$ & $\begin{array}{l}124 / 362 \\
(25.5 / 74.5)\end{array}$ & $79 / 316(20.0 / 80.0)$ & 0.05 & $49 / 154(24.1 / 75.9)$ & 0.70 & $13 / 92(12.4 / 87.6)$ & 0.004 & 7/34(17.1/82.9) & 0.23 & $4 / 11(26.7 / 73.3$ \\
\hline \multicolumn{12}{|l|}{ rs9658584 } \\
\hline \multirow[t]{2}{*}{ Allele } & G & $861(88.6)$ & $691(87.5)$ & & $357(87.9)$ & & $187(89.0)$ & & $73(89.0)$ & & $24(80.0)$ \\
\hline & $\mathrm{C}$ & $111(11.4)$ & $99(12.5)$ & 0.47 & $49(12.1)$ & 0.73 & $23(11.0)$ & 0.85 & $9(11.0)$ & 0.90 & $6(20.0)$ \\
\hline \multirow[t]{2}{*}{ Genotype } & $\mathrm{G} / \mathrm{G}$ & $381(78.4)$ & $303(76.7)$ & & $160(78.8)$ & & $82(78.1)$ & & $32(78.1)$ & & $10(66.7)$ \\
\hline & $\mathrm{C} / \mathrm{C}$ & $6(1.2)$ & $7(1.8)$ & 0.49 & $6(3.0)$ & 0.13 & $0(0)$ & 0.26 & $0(0)$ & 0.48 & $1(6.7)$ \\
\hline Dominant & $\mathrm{GC}+\mathrm{CC} / \mathrm{GG}$ & $105 / 381(21.6 / 78.4)$ & $92 / 303(23.3 / 76.7)$ & 0.55 & $43 / 160(21.2 / 78.8)$ & 0.90 & $23 / 82(21.9 / 78.1)$ & 0.95 & $9 / 32(21.9 / 78.1)$ & 0.96 & $5 / 10(33.3 / 66.7$ \\
\hline Recessive & $\mathrm{CC} / \mathrm{GC}+\mathrm{GG}$ & $6 / 480(1.2 / 98.8)$ & $7 / 388(1.8 / 98.2)$ & 0.51 & 6/197(3.0/97.0) & 0.12 & $0 / 105(0 / 100)$ & 0.25 & $0 / 41(0 / 100)$ & 0.47 & 1/14(6.7/93.3) \\
\hline
\end{tabular}

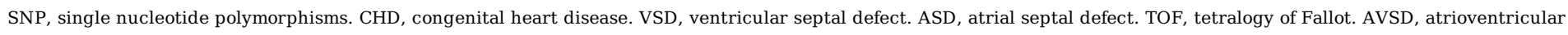
septal defect. Bold $P$-values indicate significance after Bonferroni correction.

Table 3 The risk association of CCN1 Polymorphism with ASD in Northwest Han Chinese population at different altitudes. 


\begin{tabular}{|c|c|c|c|c|c|c|c|c|c|c|c|c|c|}
\hline \multirow{2}{*}{$\begin{array}{l}\text { SNP genetic } \\
\text { model }\end{array}$} & \multirow[t]{2}{*}{ Pattern } & \multicolumn{4}{|l|}{ all } & \multicolumn{4}{|c|}{ Altitude $2500-4287 \mathrm{~m}$} & \multicolumn{4}{|c|}{ Altitude $1000-2500 \mathrm{~m}$} \\
\hline & & Control n(\%) & ASD Case $n(\%)$ & $\begin{array}{l}\text { OR } \\
(95 \% \mathrm{CI})\end{array}$ & $P$ & Control n(\%) & ASD Case n(\%) & $\begin{array}{l}\text { OR } \\
(95 \% \mathrm{CI})\end{array}$ & $P$ & Control n (\%) & ASD Case n (\%) & $\begin{array}{l}\text { OR } \\
(95 \% \mathrm{CI})\end{array}$ & $P$ \\
\hline \multicolumn{14}{|l|}{ rs954353 } \\
\hline \multirow[t]{3}{*}{ Allele } & A & $476(66)$ & $133(63)$ & & & $252(68)$ & $70(62)$ & & & $224(64)$ & $63(64)$ & & \\
\hline & G & $246(34)$ & $77(37)$ & $1.10(0.81-$ & 0.47 & $118(32)$ & $42(38)$ & $1.30(0.82-$ & 0.27 & $128(36)$ & $35(36)$ & $0.97(0.61$ - & 0.91 \\
\hline & & & & $1.50)$ & & & & $2.00)$ & & & & 1.60) & \\
\hline \multirow[t]{5}{*}{ Genotype } & $\mathrm{AA}$ & $154(42.7)$ & $43(41)$ & & & $84(45.4)$ & $22(39.3)$ & & & $70(39.8)$ & $21(42.9)$ & & \\
\hline & $\mathrm{AG}$ & $168(46.5)$ & $47(44.8)$ & $1.00(0.63-$ & 0.99 & $84(45.4)$ & $26(46.4)$ & $1.20(0.62-$ & 0.61 & $84(47.7)$ & $21(42.9)$ & $0.83(0.42-$ & 0.60 \\
\hline & & & & 1.60) & & & & 2.20) & & & & 1.60) & \\
\hline & GG & $39(10.8)$ & $15(14.3)$ & $1.40(0.69-$ & 0.25 & $17(9.2)$ & $8(14.3)$ & $1.80(0.69-$ & 0.23 & $22(12.5)$ & $7(14.3)$ & $1.10(0.40-$ & 0.91 \\
\hline & & & & $2.70)$ & & & & $4.70)$ & & & & $2.80)$ & \\
\hline \multirow[t]{2}{*}{ Dominant } & $\mathrm{AG}+\mathrm{GG} / \mathrm{AA}$ & $207 / 154(57.3 / 42.7)$ & $62 / 43(59 / 41)$ & $1.10(0.69-$ & 0.76 & $101 / 84(54.6 / 45.4)$ & $34 / 22(60.7 / 39.3)$ & $1.30(0.70-$ & 0.42 & $106 / 70(60.2 / 39.8)$ & $28 / 21(57.1 / 42.9)$ & $0.88(0.46-$ & 0.70 \\
\hline & & & & $1.70)$ & & & & 2.40) & & & & 1.70) & \\
\hline \multirow[t]{2}{*}{ Recessive } & $\mathrm{GG} / \mathrm{AG}+\mathrm{AA}$ & $39 / 322(10.8 / 89.2)$ & $15 / 90(14.3 / 85.7)$ & $1.40(0.73-$ & 0.33 & $17 / 168(9.2 / 90.8)$ & $8 / 48(14.3 / 85.7)$ & $1.60(0.67-$ & 0.27 & $22 / 154(12.5 / 87.5)$ & $7 / 42(14.3 / 85.7)$ & $1.20(0.47-$ & 0.74 \\
\hline & & & & 2.60) & & & & $4.00)$ & & & & 2.90) & \\
\hline \multicolumn{14}{|l|}{ rs3753793 } \\
\hline \multirow[t]{3}{*}{ Allele } & A & $446(62)$ & $149(71)$ & & & $220(59.0)$ & $80(71.0)$ & & & $226(64)$ & $69(70)$ & & \\
\hline & $\mathrm{C}$ & $276(38)$ & $61(29)$ & $0.66(0.47$ & 0.015 & $150(41.0)$ & $32(29.0)$ & $0.59(0.37$ & 0.022 & $126(36)$ & $29(30)$ & $0.75(0.46-$ & 0.25 \\
\hline & & & & $0.92)$ & & & & $0.93)$ & & & & $1.20)$ & \\
\hline \multirow[t]{5}{*}{ Genotype } & $\mathrm{A} / \mathrm{A}$ & $132(36.6)$ & $52(49.5)$ & & & $65(35.1)$ & $29(51.8)$ & & & $67(38.1)$ & $23(46.9)$ & & \\
\hline & $\mathrm{A} / \mathrm{C}$ & $182(50.4)$ & $45(42.9)$ & $0.63(0.40-$ & 0.045 & $90(48.6)$ & $22(39.3)$ & $0.55(0.29-$ & 0.06 & $92(52.3)$ & $23(46.9)$ & $0.73(0.38-$ & 0.34 \\
\hline & & & & $0.99)$ & & & & $1.00)$ & & & & 1.40) & \\
\hline & $\mathrm{C} / \mathrm{C}$ & $47(13)$ & $8(7.6)$ & $0.43(0.19-$ & 0.04 & $30(16.2)$ & $5(8.9)$ & $0.37(0.13-$ & 0.06 & $17(9.7)$ & $3(6.1)$ & $0.51(0.14-$ & 0.31 \\
\hline & & & & $0.98)$ & & & & 1.10) & & & & 1.90) & \\
\hline \multirow[t]{2}{*}{ Dominant } & $\mathrm{AC}+\mathrm{CC} / \mathrm{AA}$ & $229 / 132(63.4 / 36.6)$ & $53 / 52(50.5 / 49.5)$ & $0.59(0.38-$ & 0.02 & $120 / 65(64.9 / 35.1)$ & $27 / 29(48.2 / 51.8)$ & $0.50(0.28-$ & 0.03 & $109 / 67(61.9 / 38.1)$ & $26 / 23(53.1 / 46.9)$ & $0.69(0.37-$ & 0.32 \\
\hline & & & & $0.91)$ & & & & $0.92)$ & & & & 1.30) & \\
\hline \multirow[t]{2}{*}{ Recessive } & $\mathrm{CC} / \mathrm{AC}+\mathrm{AA}$ & $47 / 314(13 / 87)$ & $8 / 97(7.6 / 92.4)$ & $0.55(0.25-$ & 0.17 & $30 / 155(16.2 / 83.8)$ & $5 / 51(8.9 / 91.1)$ & $0.51(0.19-$ & 0.20 & $17 / 159(9.7 / 90.3)$ & $3 / 46(6.1 / 93.9)$ & $0.61(0.17$ & 0.58 \\
\hline & & & & $1.20)$ & & & & $1.40)$ & & & & $2.20)$ & \\
\hline
\end{tabular}




\begin{tabular}{|c|c|c|c|c|c|c|c|c|c|c|c|c|c|}
\hline \multirow[t]{3}{*}{ Allele } & G & $369(51)$ & $124(59)$ & & & $184(50)$ & $69(62)$ & & & $185(53)$ & $55(56)$ & & \\
\hline & \multirow[t]{2}{*}{ A } & \multirow[t]{2}{*}{$353(49)$} & \multirow[t]{2}{*}{$86(41)$} & $0.72(0.53-$ & \multirow[t]{2}{*}{0.05} & \multirow[t]{2}{*}{$186(50)$} & \multirow[t]{2}{*}{$43(38)$} & $0.62(0.40-$ & \multirow[t]{2}{*}{0.03} & \multirow[t]{2}{*}{$167(47)$} & \multirow[t]{2}{*}{$43(44)$} & $0.87(0.55-$ & \multirow[t]{2}{*}{0.57} \\
\hline & & & & $0.99)$ & & & & $0.95)$ & & & & $1.40)$ & \\
\hline \multirow[t]{5}{*}{ Genotype } & G/G & $94(26)$ & $32(30.5)$ & & & $50(27)$ & 19(33.9) & & & $44(25)$ & $13(26.5)$ & & \\
\hline & $\mathrm{G} / \mathrm{A}$ & $181(50.1)$ & $60(57.1)$ & $0.97(0.59-$ & 0.99 & $84(45.4)$ & $31(55.4)$ & $0.97(0.50-$ & 0.99 & $97(55.1)$ & $29(59.2)$ & $1.00(0.48-$ & 0.99 \\
\hline & & & & $1.60)$ & & & & $1.90)$ & & & & $2.10)$ & \\
\hline & $\mathrm{A} / \mathrm{A}$ & $86(23.8)$ & $13(12.4)$ & $0.44(0.22-$ & 0.03 & $51(27.6)$ & $6(10.7)$ & $0.31(0.11-$ & 0.02 & $35(19.9)$ & $7(14.3)$ & $0.68(0.24-$ & 0.61 \\
\hline & & & & $0.90)$ & & & & $0.84)$ & & & & $1.90)$ & \\
\hline \multirow[t]{2}{*}{ Dominant } & $\mathrm{GA}+\mathrm{AA} / \mathrm{GG}$ & $267 / 94(74 / 26)$ & $73 / 32(69.5 / 30.5)$ & $1.30(0.79-$ & 0.33 & $135 / 50(73 / 27)$ & $37 / 19(66.1 / 33.9)$ & $0.72(0.38-$ & 0.32 & $132 / 44(75 / 25)$ & $36 / 13(73.5 / 26.5)$ & $0.92(0.45-$ & 0.85 \\
\hline & & & & $2.10)$ & & & & 1.40) & & & & $1.90)$ & \\
\hline \multirow[t]{2}{*}{ Recessive } & $\mathrm{AA} / \mathrm{GA}+\mathrm{GG}$ & $86 / 275(23.8 / 76.2)$ & $13 / 92(12.4 / 87.6)$ & $0.45(0.24-$ & 0.0103 & $51 / 134(27.6 / 72.4)$ & $6 / 50(10.7 / 89.3)$ & $0.32(0.13-$ & 0.0112 & $35 / 141(19.9 / 80.1)$ & $7 / 42(14.3 / 85.7)$ & $0.67(0.28-$ & 0.42 \\
\hline & & & & $0.85)$ & & & & $0.78)$ & & & & $1.60)$ & \\
\hline \multicolumn{14}{|l|}{ rs9658584 } \\
\hline \multirow[t]{3}{*}{ Allele } & G & $640(89)$ & $187(89.0)$ & & & $322(87)$ & $100(89)$ & & & $318(90)$ & $87(89)$ & & \\
\hline & c & $82(11)$ & $23(11.0)$ & $0.96(0.59-$ & 0.99 & $48(13)$ & $12(11)$ & $0.81(0.41-$ & 0.63 & $34(10)$ & $11(11)$ & $1.20(0.58-$ & 0.70 \\
\hline & & & & $1.60)$ & & & & $1.60)$ & & & & $2.40)$ & \\
\hline \multirow[t]{5}{*}{ Genotype } & G/G & $282(78.1)$ & $82(78.1)$ & & & $138(74.6)$ & $44(78.6)$ & & & $144(81.8)$ & $38(77.5)$ & & \\
\hline & $\mathrm{G} / \mathrm{C}$ & $76(21.1)$ & $23(21.9)$ & $1.00(0.61-$ & 0.89 & $46(24.9)$ & $12(21.4)$ & $0.82(0.40-$ & 0.72 & $30(17.1)$ & $11(22.4)$ & $1.40(0.64-$ & 0.41 \\
\hline & & & & $1.80)$ & & & & 1.70) & & & & $3.00)$ & \\
\hline & $\mathrm{C} / \mathrm{C}$ & $3(0.8)$ & $1(0.6)$ & $1.10(0.12-$ & 0.99 & $1(0.5)$ & $0(0)$ & $1.00(0.04-$ & 0.99 & $2(1.1)$ & $0(0)$ & $0.75(0.04-$ & 0.99 \\
\hline & & & & 11.0) & & & & $26.0)$ & & & & $16.0)$ & \\
\hline \multirow[t]{2}{*}{ Dominant } & $\mathrm{GC}+\mathrm{CC} / \mathrm{GG}$ & $79 / 282(21.9 / 78.1)$ & $23 / 82(21.9 / 78.1)$ & $1.00(0.59-$ & 0.99 & $47 / 138(25.4 / 74.6)$ & $12 / 44(21.4 / 78.6)$ & $0.80(0.39-$ & 0.60 & $32 / 144(18.2 / 81.8)$ & $11 / 38(22.4 / 77.5)$ & $1.30(0.60-$ & 0.54 \\
\hline & & & & 1.70) & & & & $1.60)$ & & & & $2.80)$ & \\
\hline \multirow[t]{2}{*}{ Recessive } & $\mathrm{CC} / \mathrm{GC}+\mathrm{GG}$ & $3 / 358(0.8 / 99.2)$ & $0 / 105(0 / 100)$ & $0.49(0.03-$ & 0.99 & 1/184(0.5/99.5) & $0 / 56(0 / 100)$ & $1.10(0.04-$ & 0.99 & $2 / 174(1.1 / 98.9)$ & $0 / 49(0 / 100)$ & $0.71(0.03-$ & 0.99 \\
\hline & & & & $9.50)$ & & & & $27.0)$ & & & & 15.0) & \\
\hline
\end{tabular}

SNP, single nucleotide polymorphisms. ASD, atrial septal defect. Bold $P$-values indicate significance after Bonferroni correction.

Table 4 Analysis of the association between CCN1 Polymorphism and levels of erythrocyte parameters in Luqu Tibetan (LQT) and the Lanzhou Han (LZH) populations. 


\begin{tabular}{|c|c|c|c|c|c|c|c|}
\hline SNP & Phenotype & Genetic model & Pattern & LQT (95) & LZH (95) & $P_{1}$ & $P_{2}$ \\
\hline \multirow[t]{3}{*}{ rs3753793 } & $\mathrm{RBC}$ & Dominant & $\mathrm{AC}+\mathrm{CC} / \mathrm{AA}$ & $4.89 \pm 0.51 / 4.77 \pm 0.44$ & $4.95 \pm 0.50 / 4.85 \pm 0.48$ & 0.18 & 0.01 \\
\hline & $\mathrm{RBC}$ & HET & $\mathrm{AC} / \mathrm{AA}$ & $4.93 \pm 0.53 / 4.77 \pm 0.44$ & $4.94 \pm 0.51 / 4.85 \pm 0.48$ & 0.16 & 0.01 \\
\hline & HGB & HET & $\mathrm{AC} / \mathrm{AA}$ & $141.4 \pm 18.14 / 136.2 \pm 16.17$ & $152.8 \pm 17.61 / 148.9 \pm 20.58$ & 0.33 & 0.05 \\
\hline rs2297141 & $\mathrm{RBC}$ & Dominant & $\mathrm{GA}+\mathrm{AA} / \mathrm{GG}$ & $4.90 \pm 0.50 / 4.71 \pm 0.49$ & $4.93 \pm 0.51 / 4.88 \pm 0.41$ & 0.27 & 0.01 \\
\hline
\end{tabular}

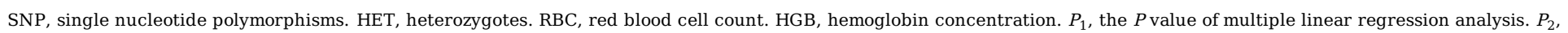
the $P$ value of multiple linear regression analysis with adjusted for age and gender.

\section{Figures}
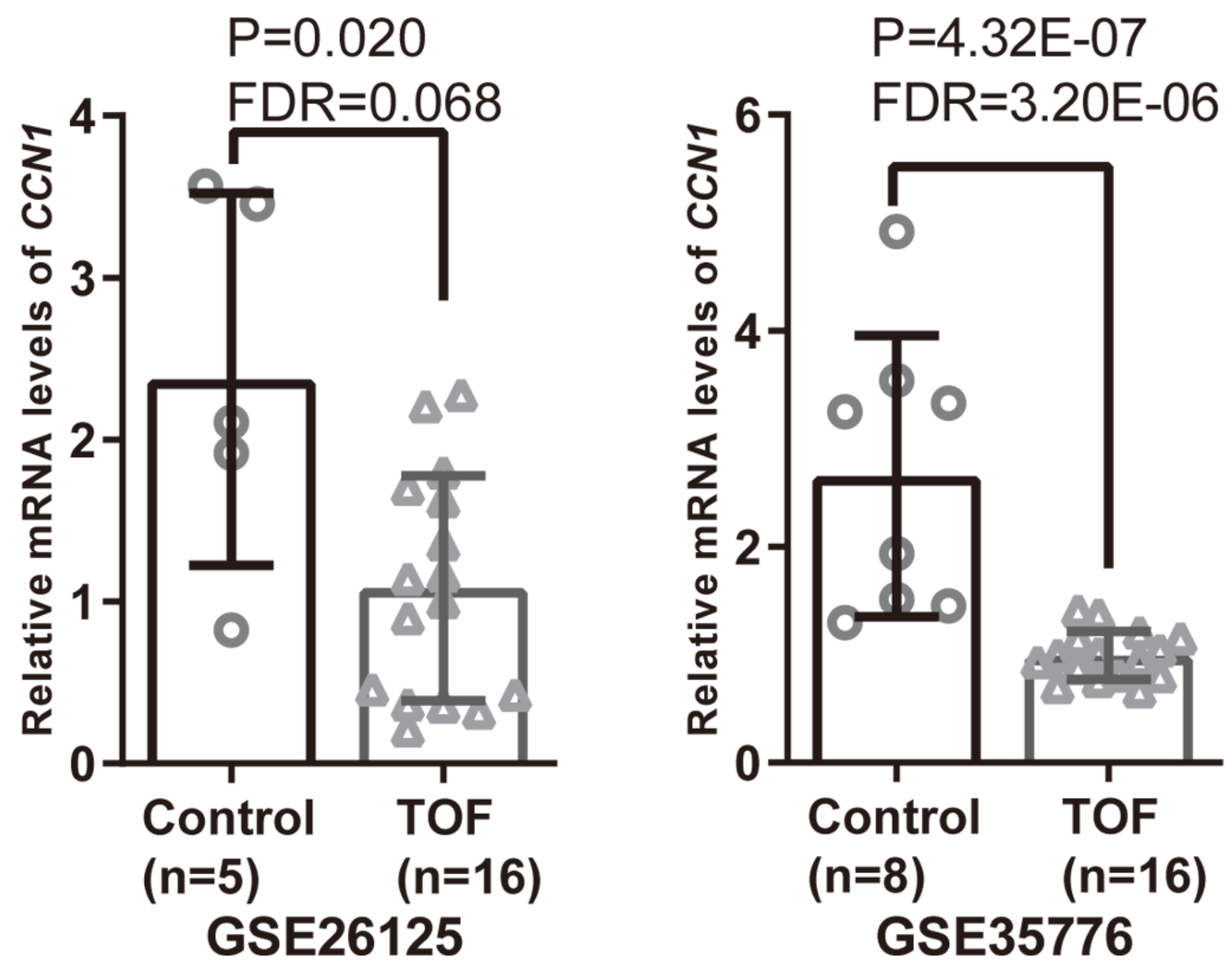

Figure 1

CCN1 mRNA expression in TOF tissues and normal tissues in GEO datasets. The CCN1 mRNA levels were significantly lower in TOFs as compared to controls. Differential expression analysis was performed using the LIMMA package with a cutoff value of $\log 2 \mathrm{FC}>1, \mathrm{P}<0.05$ and FDR $<0.10$. 

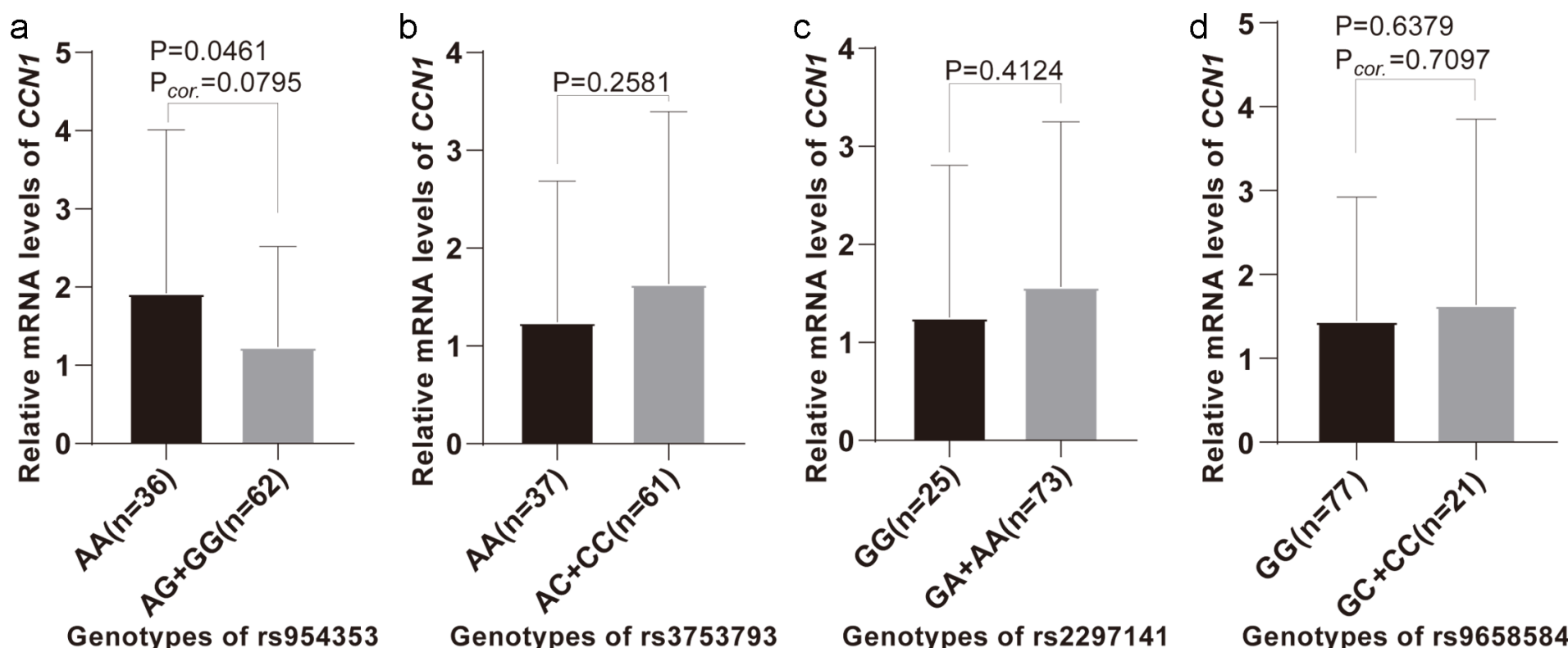

Figure 2

Relative mRNA expression levels of CCN1 in 98 myocardial tissues with different genotypes of four SNPs. (a) rs954353; (b) rs3753793; (c) rs2297141; (d) rs9658584. No association was observed between the CCN1 SNPs and CCN1 gene expression. Gene expression data were analyzed by two-tailed unpaired Student t-test for equal variances (b, c), using Welch's correction when variances were unequal $(a, d)$.

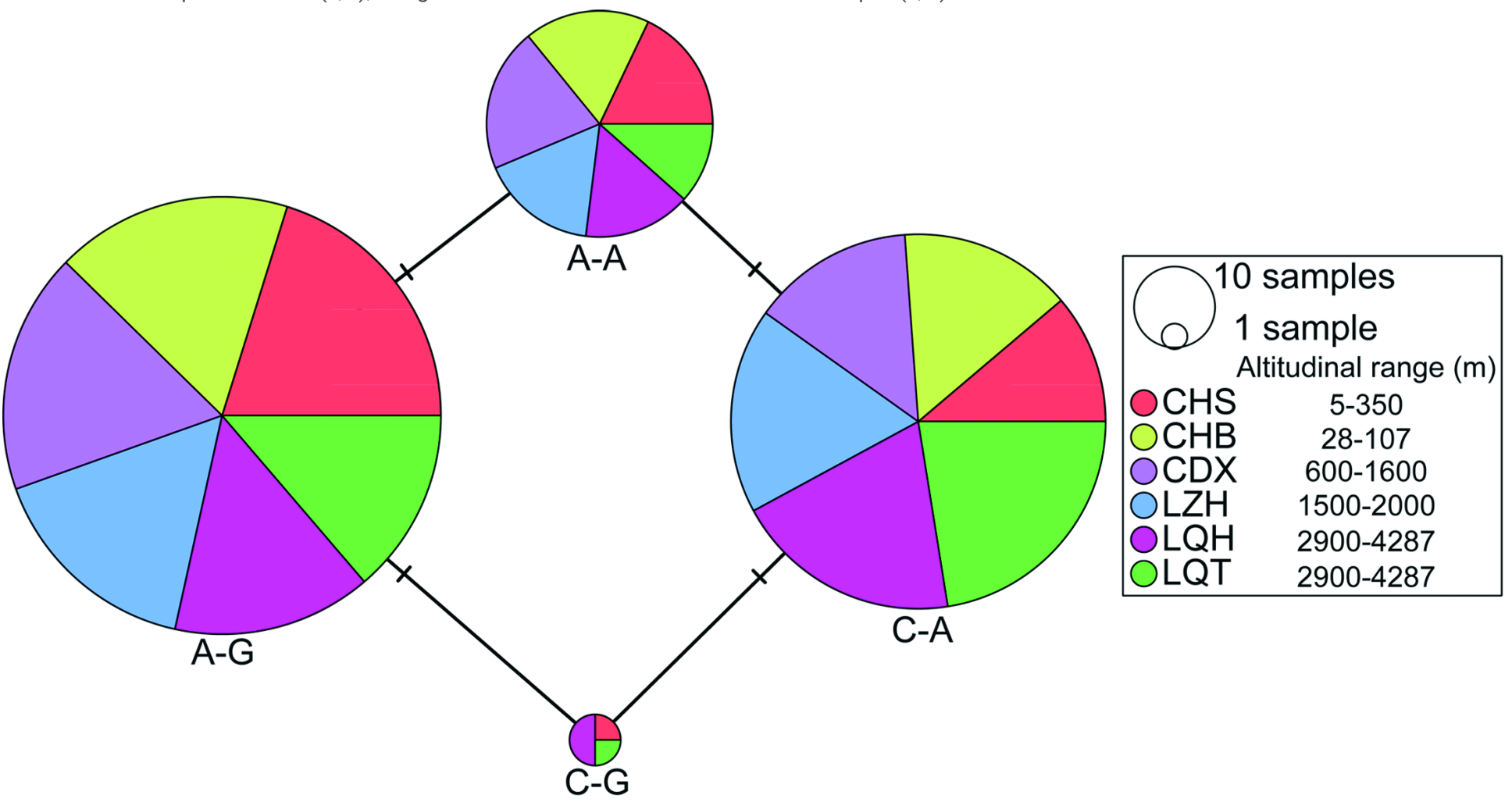

Figure 3

Network analysis of rs3753793-rs2297141 haplotypes in CHS, CHB, CDX, LZH, LQH and LQT populations at greater than $1 \%$. The protective C-A haplotype showed a high proportion in high-altitude LZH, LQH and LQT populations. Circle size is proportional to the frequency of haplotypes, segments within each circle are proportional to the haplotype frequency of the corresponding population. CHS, Southern Han Chinese. CHB, Han Chinese in Beijing. CDX, Chinese Dai in Xishuangbanna. LZH, Han Chinese in Lanzhou. LQH, Han Chinese in Luqu. LQT, Tibetan Chinese in Luqu.

\section{Supplementary Files}

This is a list of supplementary files associated with this preprint. Click to download. 
- R1tables1.docx

- R1tables2.docx

- R1tables3.docx

- R1tables4.docx

- R1tables5.docx

Page 15/15 